\title{
Towards A Proactive System for Predicting Service Quality Degradations in Next Generation of Networks based on Time Series
}

\author{
Errais Mohammed $^{1}$ \\ Research and Computer Innovation \\ Laboratory, Hassan II University of \\ Csasablanca, Casablanca-Morocco
}

\author{
Rachdi Mohamed ${ }^{2}$ \\ Faculty of Science Ben M'sik \\ Hassan II University of Casablanca \\ Casablanca-Morocco
}

\author{
Al Sarem Mohammed ${ }^{3}$ \\ Department of Information System \\ Taibah University \\ Medina-KSA
}

\author{
Abdel Hamid Mohamed Emara ${ }^{4}$ \\ Department of Computer Science, Taibah University, Medina-KSA \\ Computers and Systems Engineering Dept., Faculty of Eng., Azhar University Cairo-Egypt
}

\begin{abstract}
The architecture of Next Generation of networks (NGN) aims to diversify the offer of operators in added value services. To do this, NGN offers a heterogeneous architecture for the services deployment. This poses significant challenges in terms of end-to-end assurance of services. For this purpose, we propose in this work the establishment of a proactive autonomous system, capable of ensuring an acceptable quality level according to Service Level Agreement (SLA) requirements. A system that is able to predict any QoS degradation due to the prediction model based on time series adapted to NGN.
\end{abstract}

Keywords-Next Generation of Network (NGN); network management; enhanced Telecom Operation Management (eTOM) frameworks; prediction; time series; Ip Multimedia Subsystem (IMS); Service Level Agreement (SLA); Quality Of Service (QoS)

\section{INTRODUCTION}

Next Generation of Networks (NGN) [1] offers a standard architecture for easy integration of services with existing communication technologies. An architecture that aims primarily to facilitate the deployment and provision of valueadded services to customers of telecommunications operators [2][3]. This will help to evolve the business of the operator from a simple access provider to an end-to-end service provider.

The 3GPP [4] specifications describing the architecture of NGNs cut the network in three levels. The access level which groups together existing communication technologies. The responsible control level unifies access to networks and services regardless of the underlying technology and the level of service responsible of the unfolding and provision of valueadded services.

The diversity of communication technologies and the multitude of integrated entities pose significant challenges for end-to-end assurance of services [5]. To this end, several studies have dealt with the management of QoS in NGN networks [6][7][8]. However, most of this work proposes solutions to correct the level of the QoS after a possible degradation detected. This significantly affects the quality of the QoE user experience. Indeed, the time required for detecting and correcting degradations is very important, given the difficulty of real-time correction operations [9]. This explains the need to reduce the degradation time to ensure the satisfaction of end customers.

In this work, we propose a proactive approach to end-toend and real-time assurance of services; an approach that will predicts any degradation before sufficient time for correction. To do this we propose a prediction model able to predict the variation of the state of the network and thus to deduce the possible impairments of the QoS, in real time.

This document is organized as follows. At the beginning we will present the NGN architecture and the SLM \& M [10] solution which is used for the correction of the degradations. Then we will present the new prediction approach and discuss the model adapted to the NGN context. In the last section of the document we will discuss the results obtained during the experimentation phase in real cases of service provision.

\section{BACKGROUND AND RELATED WORKS}

\section{A. NGN Networks}

3GPP specifications dedicated to NGN networks offers a simplified architecture for the core network known by the IP Multimedia Subsystem (IMS) architecture [2]. This architecture aims to simplify the provision of service regardless of the communication technology used for service consumer access. To do this, the IMS offers unified procedures for user authentication and access to services. What is achievable by cutting the architecture at three levels is as follows:

- Access Level: This level is responsible for ensuring the connectivity of users to the control entities described in the 3GPP specifications. It allows to interface effectively with different types of communication technologies via interface components usually installed in the IMS core boundary routers. The access networks are connected to a global network linking the different control entities and the interfacing components. 
- The Control Level: This is the key level of the NGN architecture; it ensures the control of users and services deployed by the operator. The level basically includes four entities: The three control entities of the P_CSCF, I_CSCF and S_CSCF sessions and the HSS database.

- Service Level: The level that ensures the deployment of added value services. It groups logical components for negotiation with control entities as well as physical servers.

The strength of the NGN architecture lies in the ease of deployment of services without impacting the access networks deployed by the operator. However, such an organization requires the intervention of several heterogeneous entities to achieve the usual operations including user authentication and service provision. This poses challenges for managing end-toend quality of service.

\section{B. The SLM and M Solution}

The SLM\&M solution aims to automate QoS management procedures in NGN networks. An automation that has become possible through the integration of business processes of the eTOM framework [11][12]. The eTOM framework is a set of business processes designed to model the usual operations in telecommunications, in particular the provision, insurance and billing of services.

The SLM\&M solution has enabled the implementation of an autonomous system for the monitoring and correction of QoS impairments in NGN networks. This system is capable of estimating customer satisfaction in real time based on the Service Level Agreement (SLA) contract establishes when subscribing the customer to the service provided [6]. If a degradation is detected, the system proceeds to the correction based on preconfigured scenarios, as the case encountered [13].

The autonomous system resulting from the SLM\&M solution consists of several modules (Fig. 1). The modules include a set of eTOM business processes. Each process is responsible for a specific activity such as collecting metrics, loading fix configurations, and checking constraints defined in SLA.

Fig. 1 illustrates the system architecture of the SLM \& M solution which consists essentially of two levels:

- Monitoring Level: The first level includes business processes that have a global view of the network. These processes are responsible for detecting degradations and identifying configurations to correct the identified case. This level essentially includes three Services, Orchestration and Resource modules. At this level the communication between the modules is based on the SOAP protocol. A justified and validated choice [9] to facilitate the exchanges between the processes and to minimize the time of execution.

- Resource Level: The Resource Level has three modules. These modules are responsible for collecting performance indicators and implementing configurations during the correction. Communication between these modules is provided by the CORBA protocol [14]. A choice that aims to ease trade [9].

The validation of the autonomous system in real cases of service provision made it possible to focus on the limitation of the SLM \& M solution. In fact, the monitoring and correction operations make it possible to correct the QoS. However, the correction time is very important. What influences is the quality of user experience. For this purpose, the integration of technical choices to minimize time is not enough. This is explained by the difficulty of the automatic correction operation and in real time. To this end, migration to a proactive system becomes a necessity to ensure a stable level of service assurance at all times.

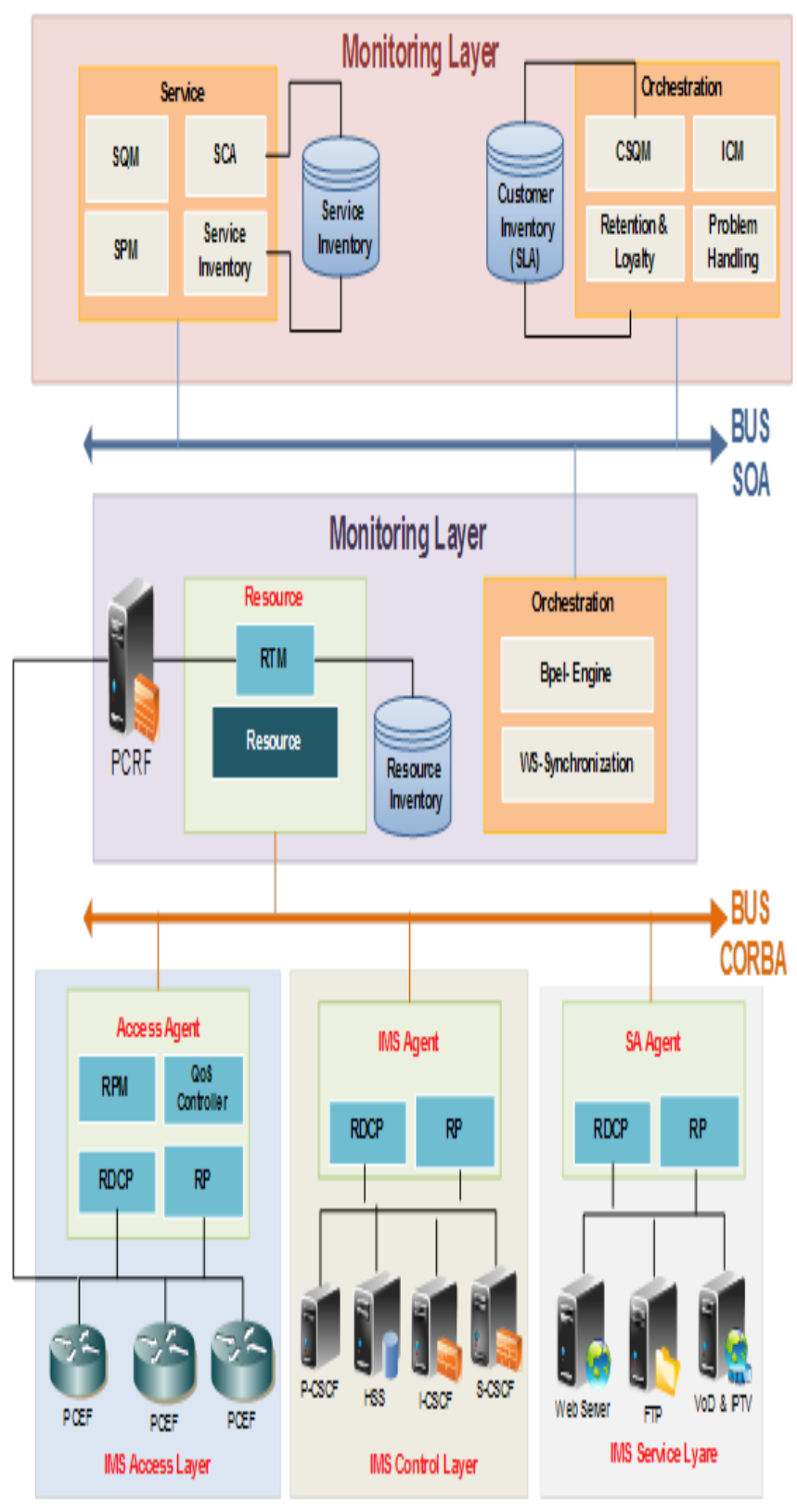

Fig. 1. System Architecture of the SLM and M Solution. 


\section{Proactive System For InSURANCE OF SERVICES}

The goal of the new approach is to migrate to a proactive system that can predict the state of the network. Once the prediction is assured the second step is to run the SLM\&M system to proceed with the implementation of the necessary configurations to avoid the degradation. Migrating the SLM \& $M$ solution to a proactive system is a difficult operation that requires multiple tasks (Fig. 2).

Fig. 2 outlines the steps required to implement the proactive approach. Steps that look like this:

- Choice of indicators to use: The choice of indicators to be estimated is a key step for the success of the approach. Indeed, the prediction operation is a difficult operation that has a major blow in terms of time and resource.

- Identification of the prediction model: There exists in the literature a multitude of mathematical techniques and models for prediction in different disciplines. The model is the key to the success of the predictive approach, given its effect on the accuracy of the predictions and thus the proactive solution.

- Integration of the model in the SLM \& M solution: After identifying the model it is necessary to interface the proactive system with the SLM \& M solution. This operation must ensure a transparent interface between the modules to ensure the proper functioning of the system.

- Testing and Validation: The final step is the validation of the evaluation of the approach in real cases of service provision.

\section{A. Choice of Performance Indicators}

Real-time monitoring is the collection of performance indicators from many resources. These indicators depend essentially on the outstanding service. For this purpose, the most fragile services are those of streaming type whose nature of flows requires regularity over time. The indicators used in this type of service belong to two categories. (i) Static indicators such as the codec used for each stream, the type of video, the type of service (VoD, IPTV) as well as the capacity of the server. (ii) Dynamic indicators such as jitter, delay, percentage of lost packets, and actual throughput.

The multitude of indicators used will undoubtedly affect the prediction time of degradation. For this, we have reduced the performance indicators used for the video stream, since it is the most sensitive to degradation. Indeed, in practical cases the degradation affects the video stream before the audio. Only the dynamic indicators will be estimated, since the static indicators remain unchanged over time. Thus, the performance indicators that will be processed in the model are (i) the jitter for the video stream, (ii) the delay for the video stream; (iii) the percentage of packets lost for the video stream.

\section{B. Choice of the Prediction Technique}

Several works have focused on the study and implementation of prediction models in different domains [15][16]. Work that offered a wide choice of models to adapt according to the domain and type of data processed. In our context, the idea is to predict future values based on the old values collected in regular time intervals. Also, the estimated values must be done in a future time are sufficient to ensure the correction operation before the actual degradation of the service.

For this purpose, time series [17] are the most appropriate technique for our context. In fact, the time series make it possible to model the values in time at regular intervals. Then these values are modeled by a mathematical model in order to be able to calculate values at times in the near future based on the values recorded in the past times.

The implementation of a prediction model based on time series requires the use of a suitable mathematical method. There are three methods in the literature for predicting values based on time series, in particular:

- Moving Average [18]: An effective method for small change values. IT is to model the series in a linear association with the moving averages of the process

- Exponential Smoothing [19]: Exponential Smoothing is a highly valued tool for predicting and analyzing data from time series observation. A technique used in industry, especially in financial markets, given the simplicity of the models included. Methods that are applicable to any discrete observation set. There are two types of smoothing: (i) simple exponential smoothing, (ii) double exponential smoothing.

- Box-Jenkins model [20]: Box-Jenkins provides an exact methodology for identifying the time series model based on recorded observations. A methodology that is based on several mathematical foundations to lead to a generally powerful prediction model.



Fig. 2. Description of the Methodology followed for the Migration to the Proactive System. 
Table I illustrates a comparison of methods for time series based on three indicators. The first is the efficiency that reflects the accuracy of the estimates from each method. The second is the cost of predicting each method while the last is the ease of adaptation of the model in the context of NGN. For this purpose, the Box-Jenkins method known also by ARMA is presented as the most efficient solution. However, setting up an ARMA-based model requires a theoretical and experimental study which cannot be done in our context. Since the nature of the flow that changes for each session, which requires the use of a model capable of correcting itself automatically. Unlike the ARMA method, exponential smoothing requires little computation, which will affect the resources less during the prediction operation. In addition, the method of smoothing has demonstrated its effectiveness in various fields. So, our choice was oriented towards exponential smoothing.

\section{Identification of the Prediction Model}

The implementation of a simple exponential smoothing prediction model consists in adapting the variable $\alpha$ (equation 1) according to the nature of the variation of the values of each indicator [19].

$$
\hat{x}_{n, t}=\alpha \sum_{j=0}^{n-1}(1-\alpha)^{j} x_{n_{-} j}
$$

The accuracy of the values estimated in the simple exponential smoothing method depends on the adjustment of the constant which takes values in the interval [0.1]. In the literature the study of the variation of the values makes it possible to adjust the constant values. However, in our context the evaluation of the values of each indicator varies according to the current session (Fig. 3). This forces the search for a selfadaptable model according to the current situation.

Fig. 3 illustrates an example of observing the delay values in three different sessions. Values that record large variations in all three sessions. This difference does not allow the use of a single constant for different sessions. Indeed, it is important to adjust the value of the constant alpha so as to minimize the error between the predicted value and the actual value (Fig. 4).

Fig. 4 illustrates the difference in the optimal value of the constant for each flow in three different sessions. This shows the difficulty of using the conventional method for identification. For this purpose, we propose a model able to justify the value of the constant according to the nature of the current session for the two indicators delay and jitter. Indeed, the experimental study showed that the value of the constant depends on the number of flows in progress in the session (Fig. 5).

In order to propose a model capable of self-justification, we propose to establish a relation between $\alpha$ and the number of flows noted ' $\mathrm{N}$ ' using linear regression [21]. Before replacing the constant by the formula in the prediction model; following this method, we obtained the following prediction models:

\section{1) Delay prediction model}

$\hat{d}_{t+h}=\alpha \sum_{j=0}^{t-1}(1-\alpha)^{j} d_{t_{-} j}$
Such as:

$\alpha=\frac{13}{10000} \cdot N+\frac{1}{4}$

With: $\mathrm{N}$ is the number of competing flows in the current session.

2) Prediction model for jitter

$\hat{g}_{t+h}=\alpha \sum_{j=0}^{t-1}(1-\alpha)^{j} g_{t_{-} j}$

Such as:

$\alpha=\frac{1}{625} \cdot N+\frac{1}{10}$

With: $\mathrm{N}$ - is the number of competing flows in the current session.

TABLE I. COMPARISON BETWEEN MODELING APPROACHES OF TIMES SERIES

\begin{tabular}{|l|l|l|l|}
\hline Method & Efficacy & $\begin{array}{l}\text { The costs of } \\
\text { implementing }\end{array}$ & Adaptability \\
\hline ARMA & $\begin{array}{l}\text { Very } \\
\text { Performance }\end{array}$ & $\begin{array}{l}\text { Costly in time and } \\
\text { resource }\end{array}$ & Very difficult \\
\hline $\begin{array}{l}\text { Exponential } \\
\text { Smoothing }\end{array}$ & Performance & Fast & Difficult \\
\hline Moving Average & Average & Fast & Easy \\
\hline
\end{tabular}
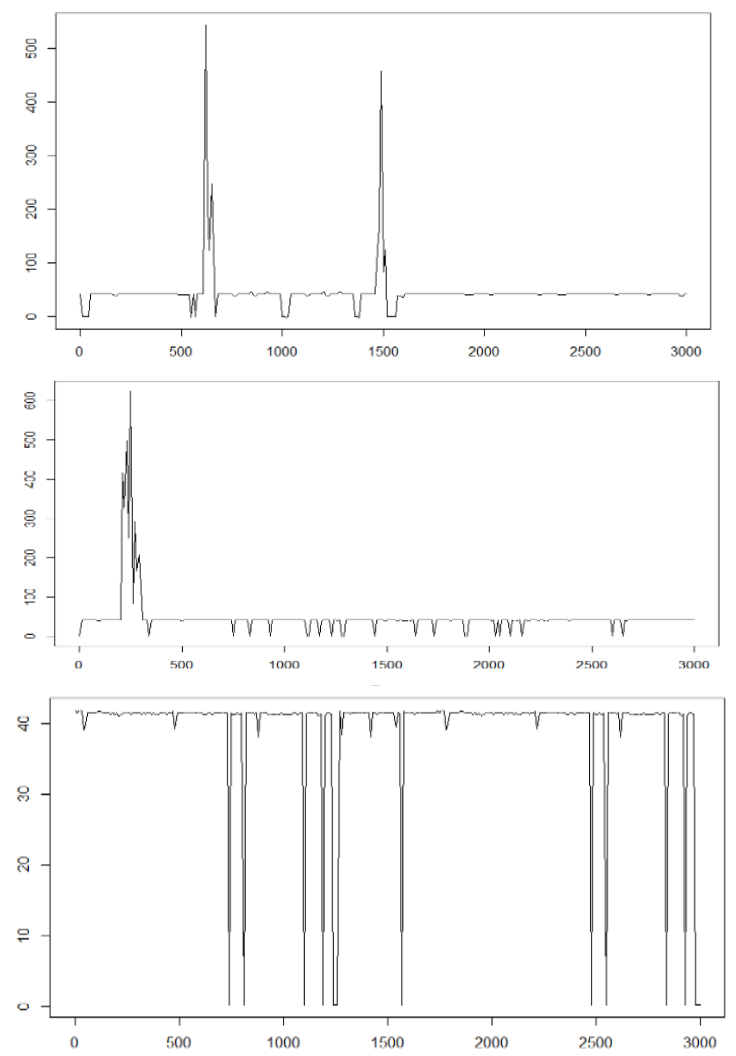

Fig. 3. Example of the Variation of the Delay Values in different Sessions. 

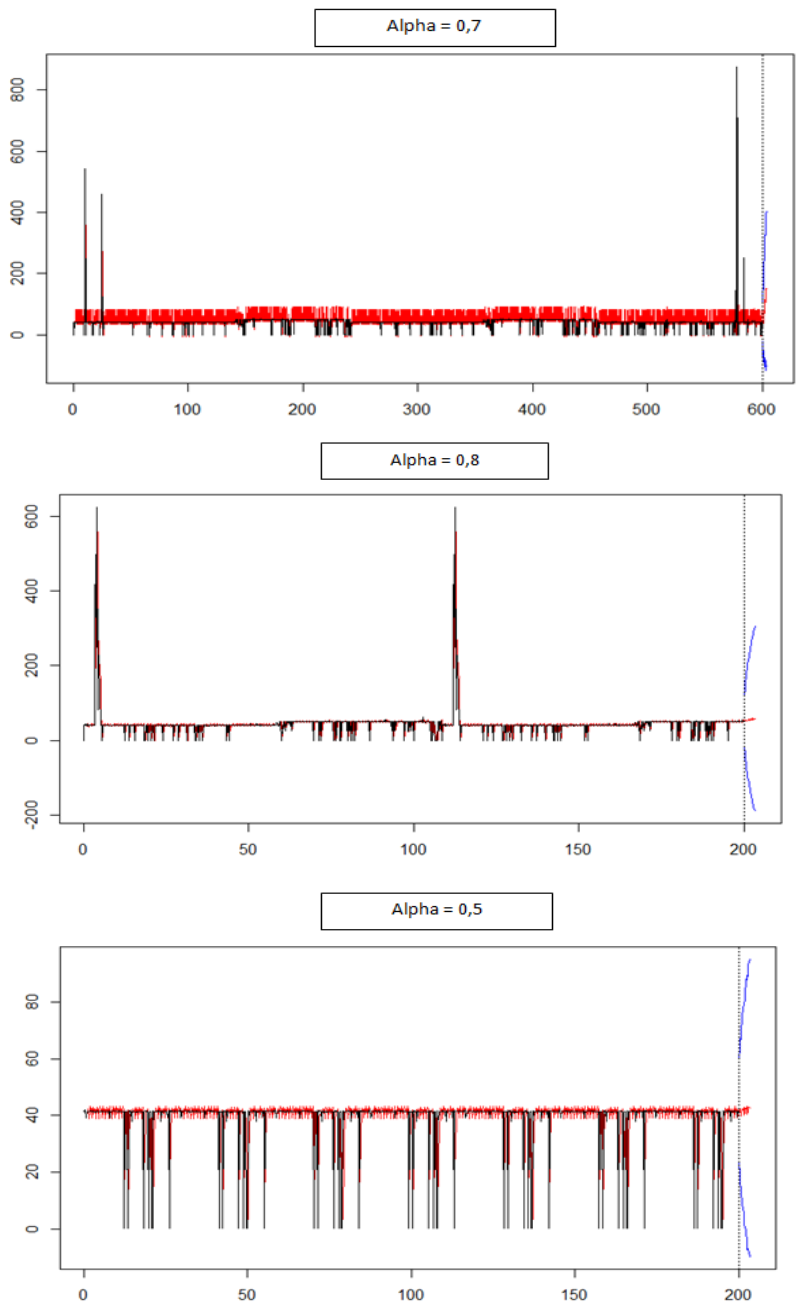

Fig. 4. Adjustment of the Constant Alpha for Three Sessions.

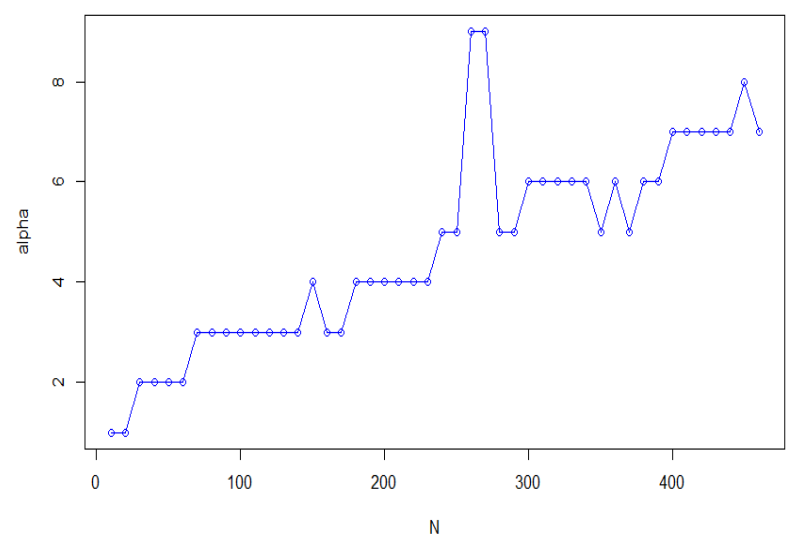

Fig. 5. The Optimal Value Variation of Alpha According to the Number of Flows.

\section{3) Model for predicting the percentage of lost packets}

Unlike the two indicators, the constant alpha does not vary significantly for the percentage of lost packets. Thus, for the model of this indicator it suffices to define the value of the constant which minimizes the margin of the error. For this purpose, the experimental study demonstrated that the most suitable value of $\alpha$ is $3 / 5$. The percentage model of packages is:

$$
\hat{P}_{t+h}=\frac{3}{5} \sum_{j=0}^{t-1}\left(\frac{12}{5}\right)^{j} P_{t_{-j}}
$$

\section{Implementation}

The integration of the proactive approach into the SLM \& $M$ system must ensure an easy and transparent exchange of messages without reducing overall system performance. The indicator estimator is developed in JAVA to facilitate integration with the existing monitoring solution (Fig. 6).

Fig. 6 illustrates the prediction system which consists of three prediction functions based on the models defined in Section III-C.

The system takes the last value collected from the Acess agent resources. These values are then saved before using them in the prediction model. The estimated values belong to a near future in order to allow the correction if a degradation is predicted. The choice to deploy the estimator directly into the resources aims to reduce the prediction time by ensuring direct communication between the indicator collection processes and the prediction system.

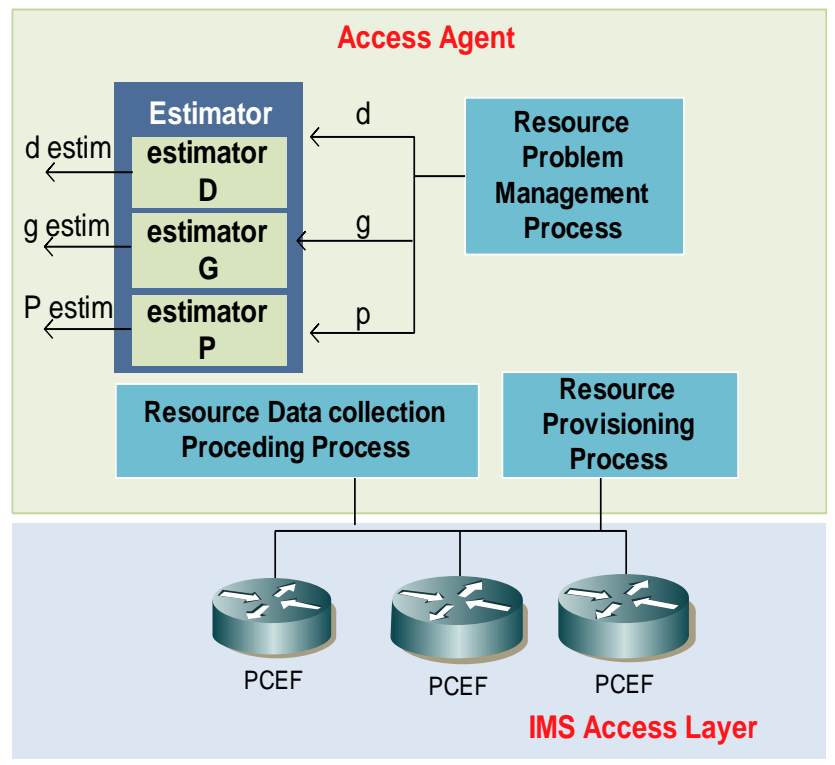

Fig. 6. Integrating the Estimator into the SLM and M Solution. 


\section{EXPERIMENTS AND RESULTS}

The experimentation phase aims at validating the prediction approach in real cases of service provision in the NGN network. In order to focus on the accuracy of the predicted values but also on the impact of such an approach on the service assurance mechanism and the execution costs on the resources. For this purpose, we propose the test bench schematized in Fig. 7 that can emulate an NGN network and the various modules of the proactive system.

The test bench consists of the following entities:

- Linux router in the boundaries that connect the core of the network to access networks. In addition, these routers also include the control entities deployed by the OpenIMScore [22] solution as well as the Acess module of the monitoring solution.

- Core-type linux router that includes other control functions as well as some module of the platform solution. This router provides connectivity with the application servers.

- Management Server that includes the modules of the solution belonging to the insurance level.

- Server Streaming application server of type VoD.

The nominal flow of the experiment is carried out according to two stages:

- Case 1: At first, the BoB client whose SLA is of the platinum type registers in the network before requesting the VoD service.

- Case 2: In a second step, several competing flows are launched in the network via the IPREF [23] solution.

In both experimental cases, the proactive solution is evaluated according to two essential criteria, namely the accuracy of predictions and the cost of deploying resources. The effect of the prediction of the indicators on the quality of the experiment is also taken into consideration during the experimental phase.

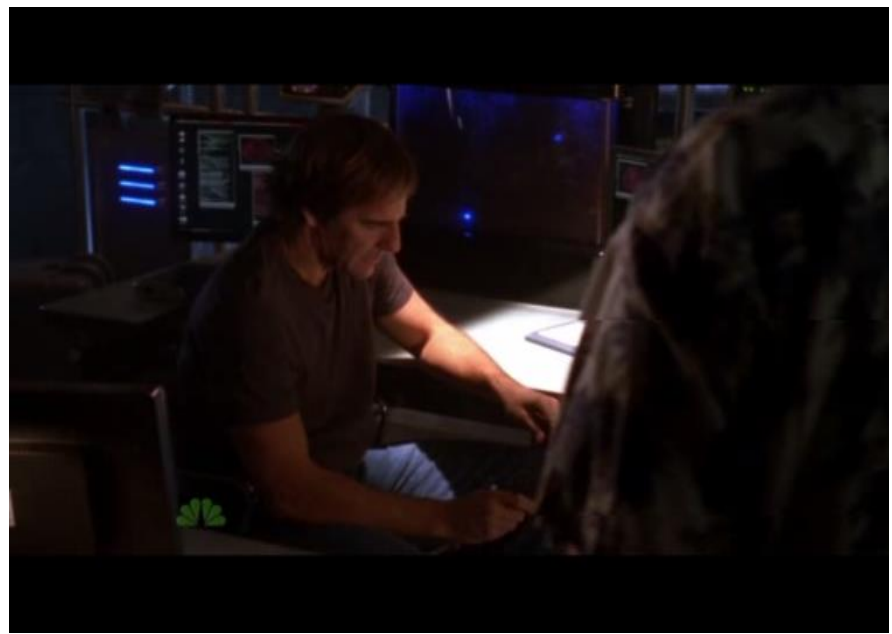

Fig. 7. Illustrates a Screenshot of the Video in the Second Experimental Case.
Fig. 8 illustrates the quality of the video captured in the first experimental case. For this purpose, the quality is very acceptable, given the absence of competing flows in the network. Thus, the SLA contract of the customer is perfectly respected by the service provider.

For this purpose, we note that the quality of video remains acceptable despite the presence of competing flows in the network. This is explained by the prediction of the indicators which made it possible to launch the configurations of the corrections in order to avoid the effective degradation of the quality of experience of the user BOB (Fig. 9).

After validation of the proactive approach it is important to evaluate the impact of the prediction on resources. Fig. 10 illustrates the rate of CPU consumption in routers according to the number of flows in the network after deployment of the prediction system. It can be noted that the prediction of the indicators does not significantly affect the resources, since a difference of less than $7 \%$ is recorded in the most critical cases (160 flows). This is explained by the choice of exponential smoothing for the implementation of the prediction model.

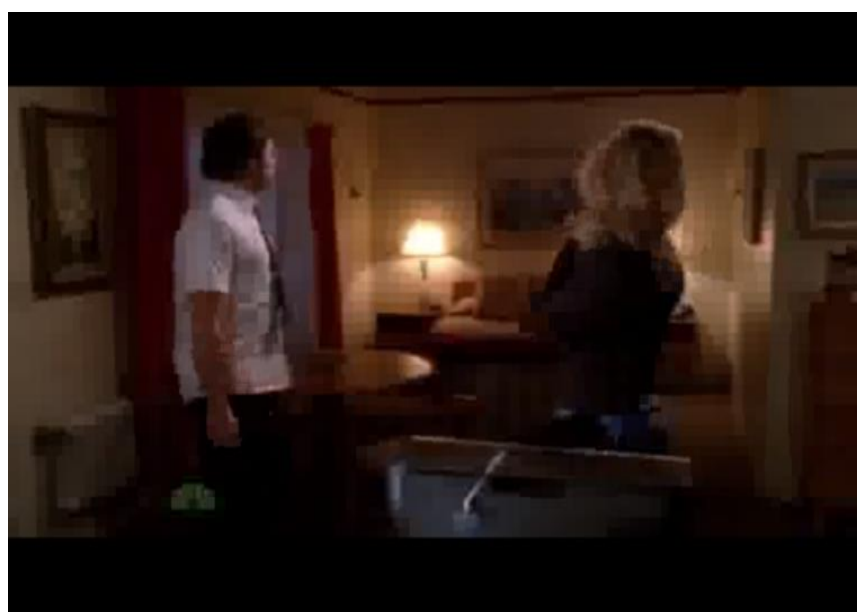

Fig. 8. Screen Video Capture in the Second Test Case.

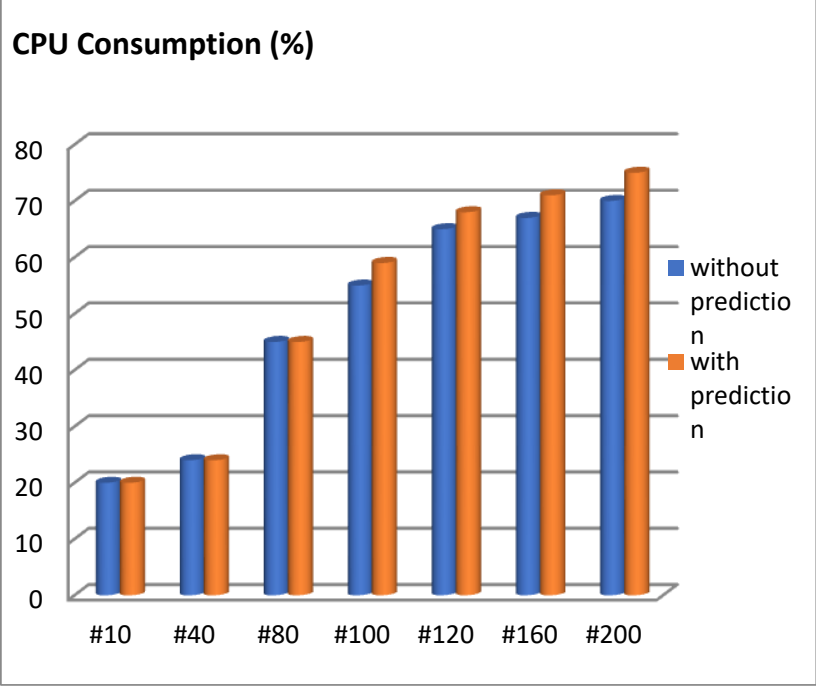

Fig. 9. CPU utilization by Routers According to the Number of Streams. 


\section{CONCLUSION}

Predicting values in the future based on observations at previous times is a complex problem that requires the use of techniques appropriate to the targeted domain. The nature of NGN monitoring, in particular the variation in observations collected, has required the adoption of an adaptive approach for the estimation of performance indicators. For this purpose, the proposed new prediction model, based on exponential smoothing and linear regression, has resulted in impeccable performance during the test phase.

The migration to a proactive and autonomous system for the monitoring and supervision of the services made NGNs highly reliable. Reliability that is shown in the level of quality of service guarantees in any moment and for different network states.

Service assurance in NGN is an important step to encourage the deployment of value-added services which is important for the diversity of the offers of telecommunication operators. However, the purchase of these services has a major blow and is not easily amortized, since services are not necessarily sold to end customers. Thus, it becomes important to think about new solutions to ensure the discovery of services directly from service providers by taking advantage of new technologies offered in this direction.

\section{REFERENCES}

[1] M. Young, The Technical Writer's Handbook. Mill Valley, CA: University Science, 1989.

[2] Maria Adamczyk, Michael Denny, Nicholas Steven, Myranda Johnson, Abdi Modarressi, Hong Nguyen, Scott Traynham ; " Application Services Infrastructure For Next Generation Networks Including A Notification Capability And Related Methods And Computer Program Products “; Patent N US 9.288,276 B2 ; 15 March 2016.

[3] Hubert Przybysz; "Andling Multiple User Interfaces In An Ip Multimedia Subsystem”; Patent N: US 8.472,376-B2 ; Jun.25, 2013.

[4] RAOUYANE Brahim, BELMEKKI Elmostafa, KHAIRI sara and BELLAFKIH mostafa, "Impact of Security in QoS Signaling in NGN: Registration Study" International Journal of Advanced Computer Science and Applications (IJACSA), 9(8), 2018.

[5] IP Multimedia Subsystem (IMS); Stage 2, 3GPP, TS 23,228, Release 9,2010 .

[6] J.L. Chen, S.L. Wuy, Y.T. Larosa, P.J. Yang, and Y.F.Li, "IMS cloud computing architecture for high-quality multimedia applications," in 2011 7th International Wireless Communications and Mobile Computing Conference, 2011, pp. 1463-1468.

[7] Bo Li, M. Hamdi, Dongyi Iang, Xi-Ren Cao and Y. T. Hou, "QoS enabled voice support in the next generation Internet: issues, existing approaches and challenges," in IEEE Communications Magazine, vol. 38, no. 4, pp. 54-61, Apr 2000. doi: 10.1109/35.833557.

[8] Youssef SERAOUI, Brahim RAOUYANE and Mostafa BELLAFKIH. "An Extended IMS Framework With a High-Performance and Scalable Distributed Storage and Computing System". The International Symposium on Networks, Computers and Communications (ISNCC) Marrakech, Morocco 16-18 May 2017.

[9] J. Zhao, H. Wang, J. Dong, and S. Cheng, "A reliable and highperformance distributed storage system for P2P-VoD service," in 2012 IEEE Int. Conf. on Cluster Computing, 2012, pp. 614-617.

[10] Mohammed Errais, Mostafa Bellafkih and Brahim Raouyane "Fuzzy Video Streaming Control in IP Multimedia Subsystem Architecture" 9th International Conference on Intelligent Systems: Theories and Applications 07-08 May 2014, Rabat, Morocco.

[11] Mohammed Errais, Mostafa Bellafkih, Daniel Ranc, "Autonomous system for network monitoring and service correction in IMS Architecture", International Journal of Computer Science \& Applications, ISSN 0972-9038, Volume 12 Issue 1, 2015.

[12] Business Process Framework (eTOM), Enhanced Telecom Operation management, GB921, version 7.2

[13] The NGOSS Real World use case, Version 1.2, GB921, T.

[14] Jon Siegel; "Corba 3 fundamentals and programming"; John Wiley \& Sons, 2000 - 899 pages.

[15] Kennedy Were, TienBu, Ystein Dick, Bal Ram Singh ; “ A comparative assessment of support vector regression, artificial neural networks, and random forests for predicting and mapping soil organic carbon stocks across an Afromontane landscape ", Ecological Indicators Volume 52, May 2015, Pages 394-403.

[16] Kay H. Brodersen, Fabian Gallusser, Jim Koehler, Nicolas Remy, and Steven L. Scott; "Inferring causal impact using Bayesian structural timeseries models"; The Annals of Applied Statistics , Volume 9, Number 1 (2015), 247-274.

[17] Erdal Kayacan, Baris Ulutas, Okyay Kaynak; "Grey system theorybased models in time series prediction"; Expert Systems with Applications Volume 37, Issue 2, March 2010, Pages 1784-1789.

[18] Charles C.Holt; "Forecasting seasonals and trends by exponentially weighted moving averages"; International Journal of Forecasting Volume 20, Issue 1, January-March 2004, Pages 5-10.

[19] Rob J. Hyndman, Anne B. Koehker, J. Keith Ord, Ralph D. Snyder ; “ Forecasting With Exponential Smoothing : The State Space Approach" BOOK; ISBN 978-3-540-71916-8.

[20] N. Garg, S. K. Mangal, P. K. Saini, P. Dhiman, S. Maji ; « Comparison of ANN and Analytical Models in Traffic Noise Modeling and Predictions" ; Acoustics Australia - Springer Link ; August 2015, Volume 43, Issue 2, pp 179-189.

[21] S. I. V Sousa, F. G. Martins, M. C. M. Alvim, Ferraz M. C. Pereira ; «Multiple linear regression and artificial neural networks based on principal components to predict ozone concentrations» ; Environmental Modelling \& Software Volume 22, Issue 1, January 2007, Pages 97-103.

[22] OpenIMSCore home page, [online] Available: htp://http://www. openimscore.org/.

[23] IPerf home page, [online] Available: https://iperffr/. 